\title{
Integrating Production and Maintenance for a Multi-Lines System
}

\author{
GHITA ETTAYE*, ABDELLAH EL BARKANY and AHMED EL KHALFI \\ Mechanical Engineering Laboratory, Faculty of Science and Techniques, Sidi Mohamed \\ Ben Abdellah University, Fez, Morocco
}

(Received on September 22, 2016, revised on November 23, 2016)

\begin{abstract}
This article discusses the issue of integrated planning of maintenance activities and production operations at the tactical level for multi-line systems with separate resources and introducing the breaking on demand constraint and that of setup time. The maintenance policy offers preventive replacements in the beginning of each cycle and minimal repairs in case of random failure. The model defined an objective function that reduces the overall cost and can simultaneously determine the optimal production plan (producing, lunching, storing and breaking costs) and the moment of replacement. The resolution is made with the mixed integer linear solver CPLEX. Then we provide a numerical example to illustrate the results and represent the economic gap between the separate and integrated planning.
\end{abstract}

Keywords: multi-line, breaking on demand, integrated planning, Minimal repair, Preventive maintenance, Setup time.

\section{Introduction}

Generally, the production planning aims at determining the quantities to be produced, the cost and production time, but practically, planners insist on the choice between several modes of manufacture. In reality, it is necessary to choose between alternative modes of production, since several production lines (or ranges); several vendors or several subcontractors are involved in manufacturing. Each of these alternatives has a production cost and different resources consumption. Indeed, the supplier may have several offers concerning the manufacture of a finished product. These offers are different in terms of production costs as well as resource consumption and can be modeled by ranges. On the flip side, production can be local in the company or with an external subcontractor. Generally, costs vary according to subcontractors, and then we can say that the choice between several suppliers is the same as making a selection between several production lines.

In the industry we manage to find two types of production lines, those with separate resources and the other with one or more common resources.

In the case of the first situation, the finished products have completely separate production lines. In fact the, production of a product does not directly affect the production of substitutes. This configuration is shown schematically in Figure 1 which shows an example of three production lines with separate resources.

"Corresponding author's email:ghita.ettaye@usmba.ac.ma 
The second situation is more complicated. Indeed, the finished products go through one or more common resources, this is the case met in a company that has several production lines with shared resources which are often human resource and material resource. Therefore, production of a product directly affects the production of finished products. This configuration is shown schematically in Figure 2, which shows three production lines that share both resources.

In the present study we addressed the issue of lot-sizing and multi-range problem and we have considered the case of separate resources by extending the work made in [1] and those of [2] as illustrated in Figure 3.

To model this situation we are based on the model of [3] and we have considered a new dimension range (for each line) that we will denoted by g, the number of ranges is $\mathrm{G}$. products are considered distinct during the production phase but upon their entry into stock and in case of breaking on the dem²and, quantities of all equivalent products are added to meet customer requirements.

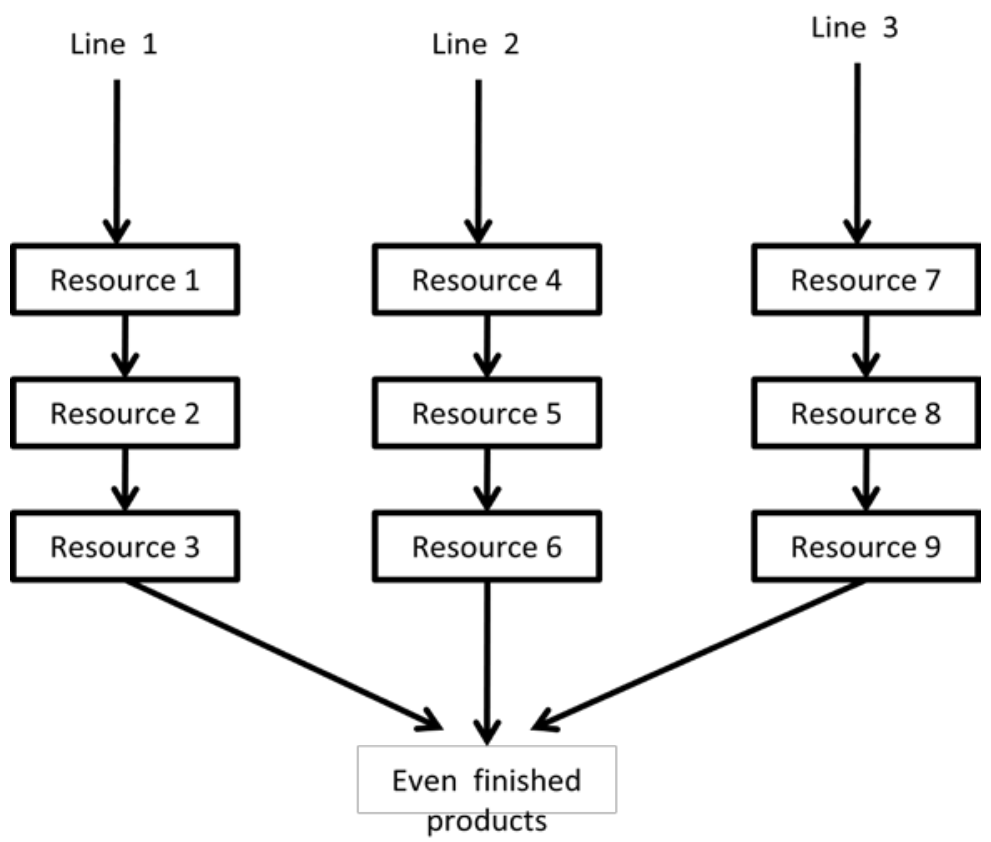

Fig. 1: Configuration of Three Lines System with Separate Resources 


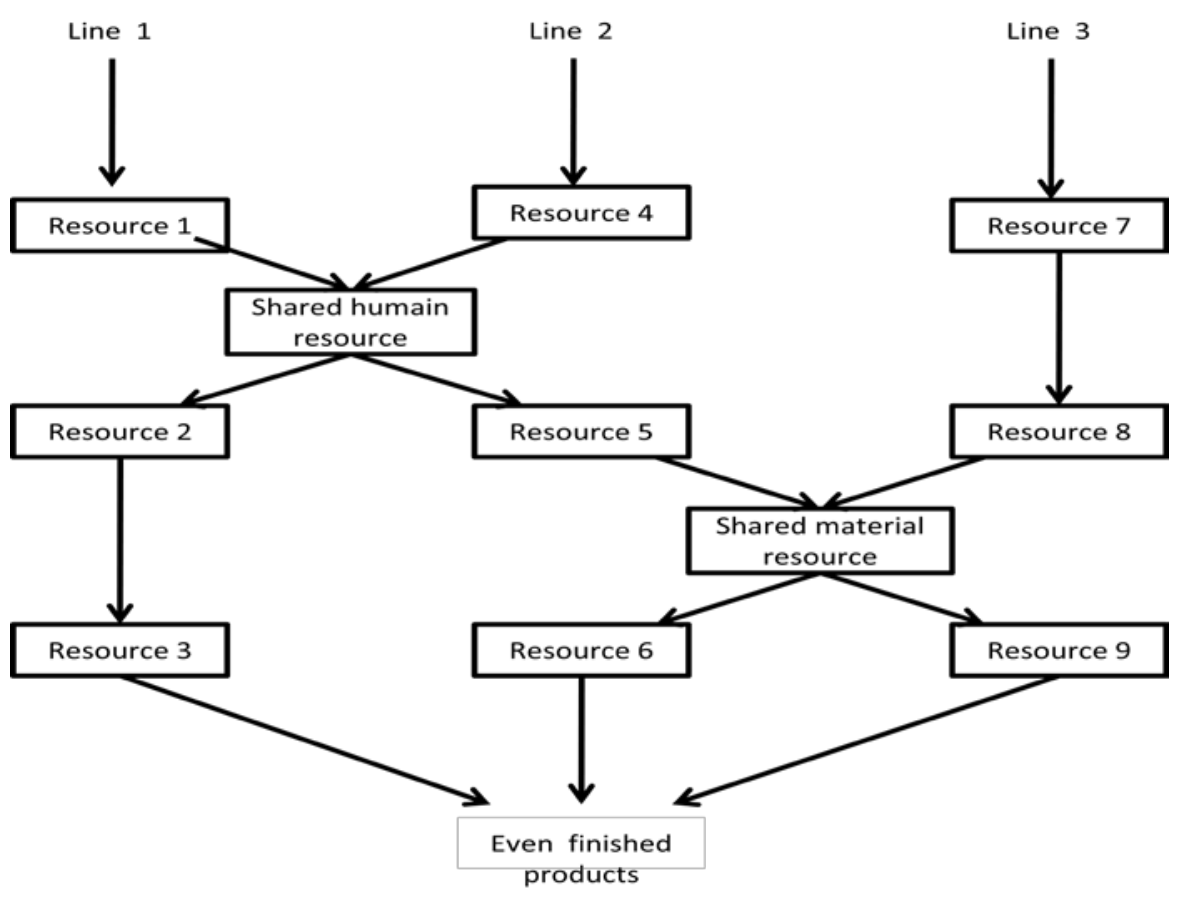

Fig. 2: Configuration of Three Lines System with Shared Resources

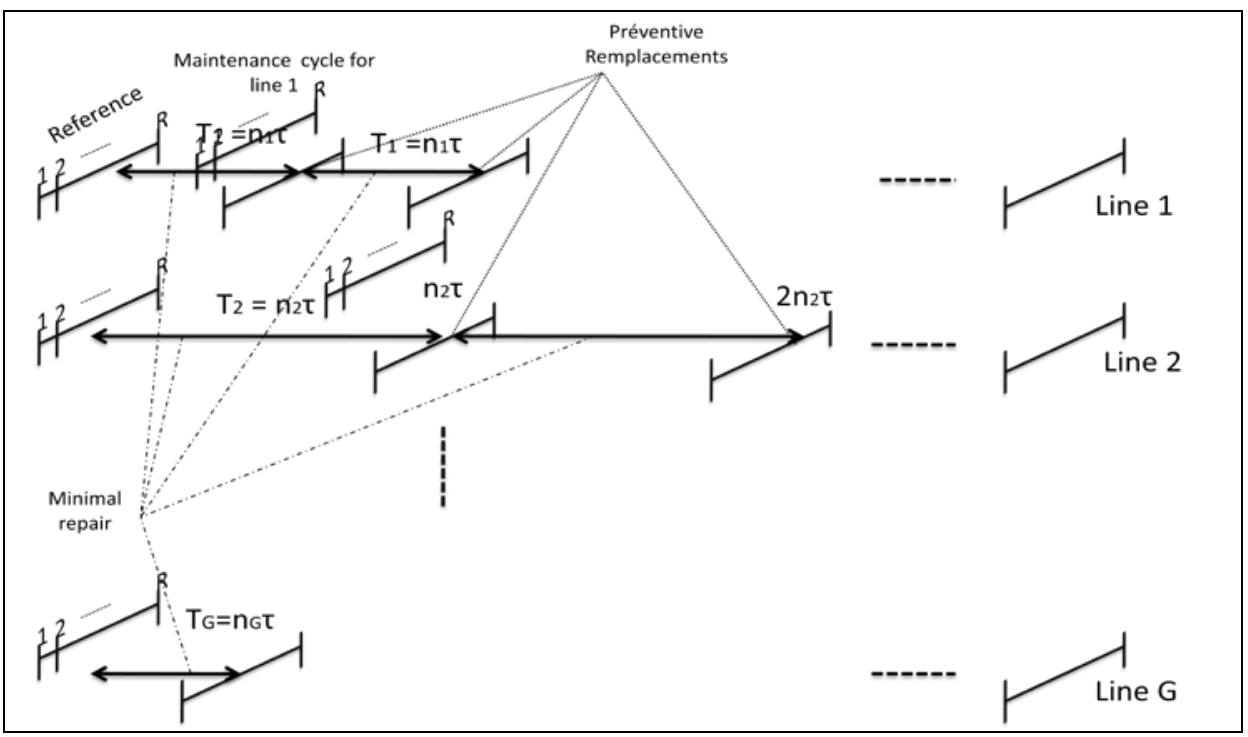

Fig. 3: Configuration of Multi-Lines Production Systems 


\section{Literature review}

Studies investigating the issue of integrating production and maintenance have shown some very promising results. However, most of these studies are dedicated to the integration of maintenance and production planning at the operational level and only few tackled the problem at an aggregate level. Many researches related to integrated planning of production and maintenance can be found in the literature during the recent years in [4] using different approaches, resolution methods, and modeling tools. Such as the work of [5] which studies the integration of maintenance and hierarchical production planning and propose a three-phase approach.

The authors of [6] propose a basic model for single machine production systems. This is the first work that offers a truly integrated planning. Unlike [5], they model both maintenance planning and production planning simultaneously. They propose a solution approach, which is based on a periodic policy (the cycle size of maintenance period is fixed). An illustrative example shows that solving production planning and maintenance planning independently may be feasible but not optimal. In [7] and [1], the model is extended to production systems with several production lines. A general preventive maintenance policy is proposed and used as a lower bound to test a Lagrangian heuristic. The general policy relaxes the cyclical restriction and establishes - for each production line - specific maintenance periods, which do not necessarily fall at equally distant epochs. The solution gap analysis shows that capacity tightness has a great impact on the quality of the Lagrangian heuristic. When capacity is loose, the heuristic produces feasible near-optimal integrated plans (gap is approximately 2 per cent). Eventually it comes integrated planning in [2] work which has not only considered the case of multi-periods, multi-lines and multi-products systems but it also took into consideration the deterioration of production lines, this degradation is represented in the model as a reduction of capacity of production lines according to the changing times, maintenance operations are supposed to provide the lines in a operating condition as good as new with maximum capacity, Through the study boundaries model, it was demonstrated that the proposed approach can deal with a larger range of problems than [1].

On the other side, it comes the work in [8], which have also used the works of [9] and [6] to study systems with parallel components considering economic and stochastic (failure of common causes FCC) dependences, they used the b-factor model to represent the FCC assuming two possible causes for system failure: the independent failure of the single components and the simultaneous FCC of all components. A maintenance policy of the T-age group was applied and in this case the components are together cyclically renewed with minimal repairs for unplanned failures by adding lot-sizing planning in order to minimize the sum of costs for a demand of products in a finite horizon. All that models pass over the breaking on demand and set up time constraints which are taken in [3]. In fact, our contribution aims to integrate the production operations and the maintenance activities in order to minimize the total cost, considering a set of constraints added to the already existing research such as the breaking on the demand, the setup time and applying a new method for solving the mixed integer linear programming problem compared to that found in the literature for multi-lines production system. 


\section{The proposed mathematical model}

\subsection{The production cost and the capacity expression}

Our paper consist of determining a production plan for a set of references $\mathrm{R}$, in a planning horizon composed of $\mathrm{N}$ long periods and for a set of lines G. To express the production cost for a multi-lines system we have used the case of single line shown in [3] which worked on the modeling of classic lot-sizing problem with finite capacity (denoted MCLS: Multi-item capacitated lot-sizing problem with setup times and Shortage costs) by introducing the cost of breaking on demand for a planning horizon composed of $\mathrm{N}$ long periods and a set of references $\mathrm{R}$. Then we use the summation of production and storage variable costs, as well as the launching and breaking on demand fixed costs in the following equation with $n_{g}$ which is the number of basic periods in the maintenance cycle by line g:

$$
C P\left(\mathrm{n}_{\mathrm{g}}\right)=\sum_{g=1}^{G} \sum_{i=1}^{R} \sum_{t=1}^{H}\left(\alpha_{g_{i t}} x_{g_{i t}}+\beta_{g_{i t}} y_{g_{i t}}\right)+\sum_{i=1}^{R} \sum_{t=1}^{H}\left(\gamma_{i t} s_{i t}+\varphi_{i t} r_{i t}\right) ; \forall g \in G ; \forall i \in R ; \forall t \in H
$$

Capacity is the theoretical maximum quantity of products that can be performed by a given operating system during a specific period of time in predetermined operating conditions. Since our case study is to plan the two Activities maintenance and production to define the available capacity of the line $\mathrm{g}$ in period $\mathrm{t}$. It is assumed that each maintenance operation consumes a percentage of the maximum capacity respectively $P p_{g}=a_{g} C \max _{g}$ for the preventive case and $P c_{g}=b_{g} C \max _{g}$ for the corrective case, knowing that $0 \leq a_{g} \leq b_{g} \leq 1$ this inequality can be explained by the fact that in case of random failure capacity resources are consumed more because some offline repair activities must in this case be achieved online.

We suppose for a given period $t \in H$ that the probability density function $\mathrm{f}_{\mathrm{g}}(\mathrm{t})$ and the cumulative distribution function $\mathrm{F}_{\mathrm{g}}(\mathrm{t})$ are known and defined for a probability distribution (law) in each line g. The failure rate of a system at period $t$ is given by the Eq. 2 and the lifetime of production line follows a probability law. The failure rate in case of Gamma and Weibull law is defined with Eq. 3 for $t \in[0, m]$.

$$
\begin{aligned}
& \lambda(t)=\frac{f(t)}{1-F(t)} ; \forall t \in H \\
& \int_{0}^{m} \lambda(t) d t=\int_{0}^{m} \frac{f(t)}{1-F(t)} d t=\log \left(\frac{1-F(0)}{1-F(m)}\right)=-\log (1-F(m)) \\
& \text { with } F(0)=0
\end{aligned}
$$

Based on the demonstration made in [2] and [3] the capacity expression is done if $t=\left(n_{g} k+u\right) \tau / k \in\left\{1, \ldots,\left[N / n_{g}\right]\right\} / u \in\left\{1, \ldots, n_{g}\right\}$ : with $C_{g}(t)=C_{g}(u \tau)$ :

Then $C_{g}(t)$ is equal to: 


$$
\left\{\begin{array}{l}
C_{g}(\tau)=C \max _{g}-P p_{g}+P C_{g} \log \left(1-F_{g}(\tau)\right) \text { if } n=1^{*} \\
C_{g}(n \tau)=C \max _{g}-P C_{g} \log \left(\frac{1-F_{g}((\mathrm{n}-1) \tau)}{1-F_{g}(\mathrm{n} \tau)}\right) \text { with }\left(n=2, \ldots, n_{g}-1\right)^{* *} \\
C_{g}\left(t=n_{g} k \tau+u \tau\right)=C_{g}(u \tau) ; \\
\text { with } k \in\left\{1, \ldots,\left[N / \mathrm{n}_{g}\right]\right\} ; u \leq\left(n_{g}-1\right) \tau ; u \leq N-k n_{g} \tau
\end{array}\right.
$$

If we know the maintenance cycle $T_{g}=n_{g} \tau$ and the probability distribution, we can calculate the capacity $C_{g t}$. We assume that capacity values are identically distributed on all the maintenance cycles $T_{g}, 2 T_{g}, 3 T_{g}, \ldots$ and for each line g. Then we calculate the capacity of the first cycle and the line g. After that we can deduce the others values $\forall t \in H ; \forall g \in G$.

\subsection{The maintenance policy and cost expression}

For a finite horizon, the Production line is degrading with time and use; so the reliability and availability of production system decrease and the failure rate increase, which influences the capacity and productivity of the line, for this reason we must choose a maintenance policy to solve all these issues. Mainly, maintenance of an industrial system is divided into two main types based on the criteria of failure: The corrective maintenance which is carried out after the failure of a system or part of the system in order to hand it to a state that allows it to perform the function assigned to it, then the preventive maintenance which is carried out periodically to ensure the proper operation and prevent failure. In our model the maintenance policy proposed to replace preventively and periodically production system at predetermined times $T_{g}=\mathrm{n}_{\mathrm{g}} t, 2 \mathrm{n}_{\mathrm{g}} t, 3 \mathrm{n}_{\mathrm{g}} t \ldots$ and to do minimal repair in case of unplanned failure inside the cycle $T_{g}$.

According to the work of [3], the total maintenance cost of a production system with one line is a nonlinear function which includes corrective cost $c c g$ for each component failed and preventive cost $c p_{g}$ for the not defaulting component; it is expressed with the following equation:

$$
C M(t)=\frac{H}{T}\left(C_{p}+c_{c} \int_{0}^{T} \lambda(t) d t\right) \quad \forall t \in H
$$

On the other hand, by adding the dimension g to the proposed model in [9] and using Eq.2, we can describe the total maintenance cost by lines $C M_{g}\left(\mathrm{n}_{\mathrm{g}}\right)$ through the following equation, where $\mathrm{N}$ is the number of basic periods in the planning horizon $\mathrm{H}$ :

$C M_{g}\left(\mathrm{n}_{\mathrm{g}}\right)=\left[N / n_{g}\right] c p_{g}-c c_{g} \log \left[\left(1-F_{g}\left(n_{g} \tau\right)\right)^{\left[N / \mathrm{n}_{\mathrm{g}}\right]}\left(1-F_{g}\left(\left(N-\left[N / n_{g}\right] n_{g}\right) \tau\right)\right)\right]$

with $T_{g}=n_{g} \tau$

In the majority of cases, the production planning horizon $\mathrm{H}$ is not a multiple of preventive maintenance cycle, if we have $\mathrm{T}_{\mathrm{g}}$ for each line $g \in G$ and we know the 
corrective cost $c c_{g}$ and the preventive cost $c p_{g}$ for each line g, as well as the probability distribution of failures we can express the value of the total maintenance cost in function of the number of periods per line $n_{g}$.

\subsection{Model formulation}

With the addition of the amendments to the parameters and the decision variables of multi-item capacitated lot-sizing with breaking on demand and setup time problem, our new model is obtained for the multi-line case. In what follows we present the data model.

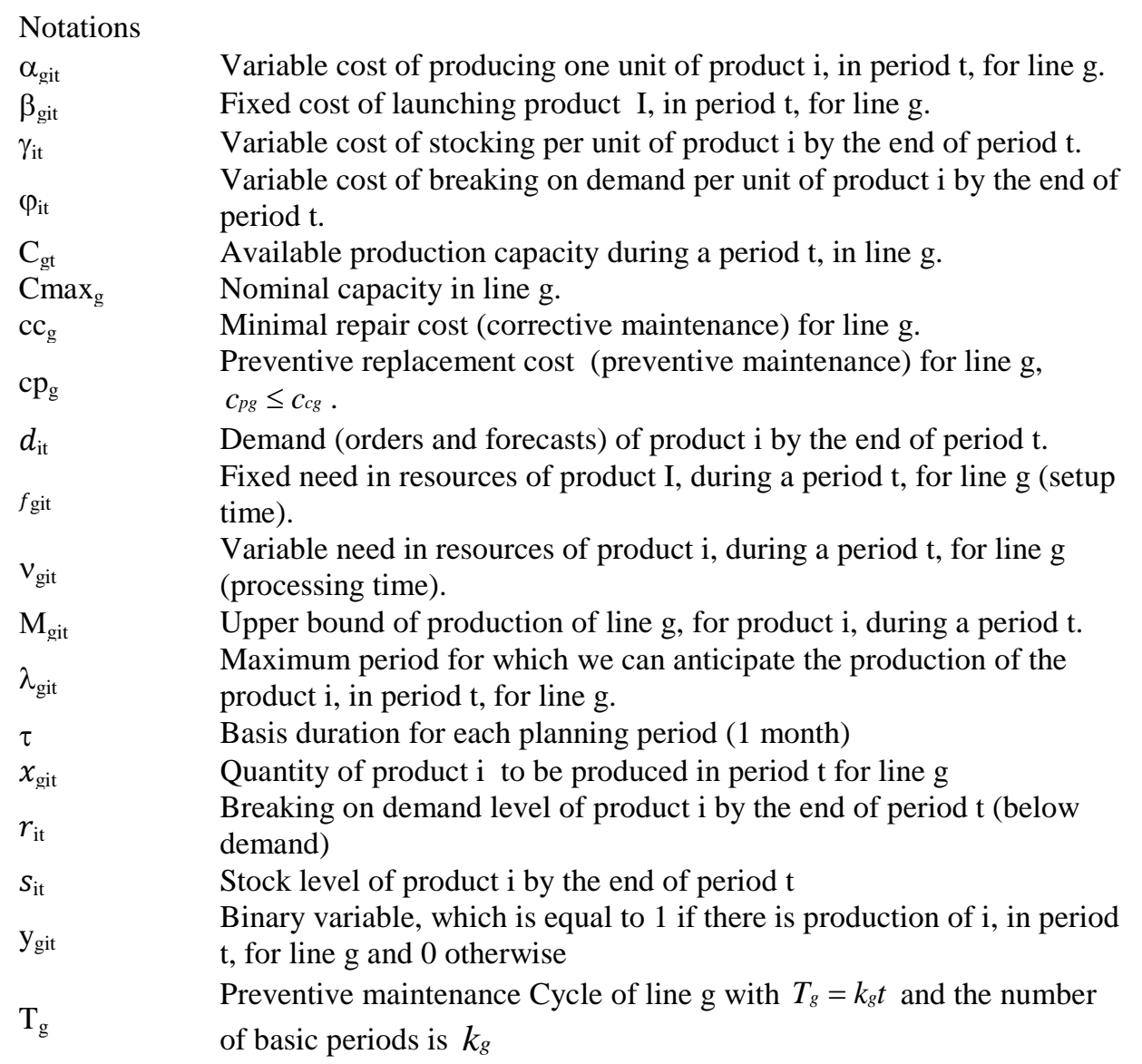

Using these variables and parameters, we express the multi-lines maintenance and production integrated planning MMPIP problem as follows: 


$$
\begin{aligned}
& \min \left(\sum_{g=1}^{G} \sum_{i=1}^{R} \sum_{t=1}^{H}\left(\alpha_{g i t} X_{g i t}+\beta_{g i t} y_{g i t}\right)+\sum_{i=1}^{R} \sum_{t=1}^{H}\left(\gamma_{i t} s_{i t}+\varphi_{i t} r_{i t}\right)+\sum_{g=1}^{G}\left(C M_{g}\left(\mathrm{ng}_{\mathrm{g}}\right)\right)\right) \\
& \text { subject to: } \\
& \sum_{g=1}^{G} X_{g i t}+r_{i t}-S_{i t}+S_{i}(t-1)=d_{i t} ; \forall i \in R, \forall t \in H \\
& \sum_{g=1}^{G} \sum_{i=1}^{R}\left(v_{\text {git }}{ }_{\text {git }}+f_{\text {git }}{ }{ }_{\text {git }}\right) \leq C_{g}(t) ; \forall g \in G ; \forall t \in H \\
& \chi_{\text {git }} \leq M_{\text {git }} * y_{g i t} ; \forall g \in G ; \forall i \in R ; \forall t \in H \\
& r_{i t} \leq d_{i t} ; \forall i \in R, \forall t \in H \\
& \sum_{g=1}^{G} y_{g i t} \leq 1 ; \forall i \in R, \forall t \in H \\
& \left\{\begin{array}{l}
x_{g i t} \geq 0 ; \forall g \in G ; \forall i \in R ; \forall t \in H \\
S_{i t}, r_{i t} \geq 0 ; \forall i \in R ; \forall t \in H
\end{array}\right. \\
& y_{\text {git }} \in\{0,1\} ; \forall g \in G ; \forall i \in R ; \forall t \in H \\
& \text { Si } 0=0 ; \forall i \in R
\end{aligned}
$$

The objective function (Eq.7) minimizes the overall Cost that comprises the production plan (producing, lunching, storing and breaking costs) and the maintenance plan (preventive and corrective tasks costs) for each line of production. Constraint (Eq. 8) shows the conservation of flows through the planning horizon for product $i$ in period $t$, it ensures that the available inventory of the product $\mathrm{i}$ increase with the quantity produced by each line to satisfy demand in the period $t$ and the rest is stored for subsequent periods (breaking). Constraint (Eq. 9) reflects the fact that the plan that we want to calculate must be with a finite capacity in all lines. Indeed, for achieving a planning, we have a quantity of resources that will be consumed by the production of one or more products and by line. The total consumption must remain less than the available capacity $\mathrm{C}_{\mathrm{gt}}$ which is expressed in each period and line by the expression in Eq. 2. Constraint (Eq. 10) indicates that if there is a production launching then the quantity produced must not exceed the upper bound of production for each line (Eq. 16). The latter is defined by the minimum between the maximum quantity of product can be produced and the demand on a segment of the horizon $\left[\mathrm{t}, \ldots, \lambda_{\text {git }}\right]$.

$$
\mathrm{M}=\min \left(\sum_{t^{\prime}=t}^{\lambda_{\mathrm{git}}=N \tau} d_{i t^{\prime}} ;\left(\left(C_{g t}-f_{g i t}\right) / v_{g i t}\right)\right) ; \forall g \in G ; \forall i \in R ; \forall t \in T_{j}
$$

Constraint (Eq. 11) ensures that the breaking on demand for product $\mathrm{i}$ by the end of period $t$ is less than the demand for the same product to the same period. At any moment, each product is planned at most a single line for constraint (Eq.12). Constraint (Eq. 13) assures that the variables $x_{\text {git }}, s_{i t}$ and $r_{i t}$ are continuous positive for any reference $i$, in each period $t$ and for each line g. Constraint (Eq. 14) expresses the fact that $\mathrm{y}_{\text {git }}$ is a dependent 
binary variable of production for any product $i$, each period $t$ and for every. Finally, the constraint (Eq. 15) expresses that initial inventory for each product $i$ which is void at $\mathrm{t}=0$.

\section{Problem resolution}

To determine the optimal integrated production and maintenance plan for multi-line degraded production system we used the following procedure with the following set of tasks:

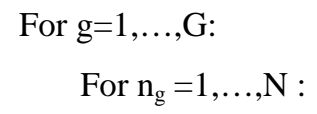

- Task 1: Calculate the maintenance costs $\mathrm{CM}_{\mathrm{g}}\left(\mathrm{n}_{\mathrm{g}}\right)$ : in this case the optimal maintenance cycle for line $\mathrm{g}\left(\mathrm{T}_{\mathrm{g}}{ }^{*}=\mathrm{n}_{\mathrm{g}}{ }^{*} \tau\right)$ is that of the minimal value of $\mathrm{CM}_{\mathrm{g}}\left(\mathrm{n}_{\mathrm{g}}\right)$.

- Task 2: Calculate the capacity values Cgt for each period $t \in H$ and each line $g \in G$.

- Task 3: Apply the mixed integer linear programming with CPLEX for each line $g \in G$ to solve the multi-lines MCLS (with set up time and breaking on demand) problem in order to determine the production costs by using the obtained values of capacity Cgt.

- Task 4:find the total costs MMPIP (ng), and select the optimal maintenance cycle $\mathrm{Tg}^{*}$ which is equal to min $\{$ MMPIP (ng)\}.

- Deduce the final solution by expressing the variable values Tg, xgit, ygit, sit, rit.

The proposed solution algorithm (see the following flowchart in Fig 4) is coded using Matlab R2015a and ILOG OPL ICPLEX 12.6. Then, all digital work has been made in a computer micro processor core i5, 5th generation with 4 GB of RAM processor.

\section{Case study}

To illustrate the advantages of integrating simultaneously the maintenance activities and the production operations in multi-lines production system considering the setup time and the breaking on demand constraints, we propose to study a system with a planning horizon $H=11 \tau$, such as the basic production period $\tau=1$ month and the number of periods is $N=11$. We consider also two products $R=2$ in lots to be produced during this horizon and two production lines $G=2$ (two different ranges) to satisfy the client demands which are shown in Table 1 for each period $t \in H$. 


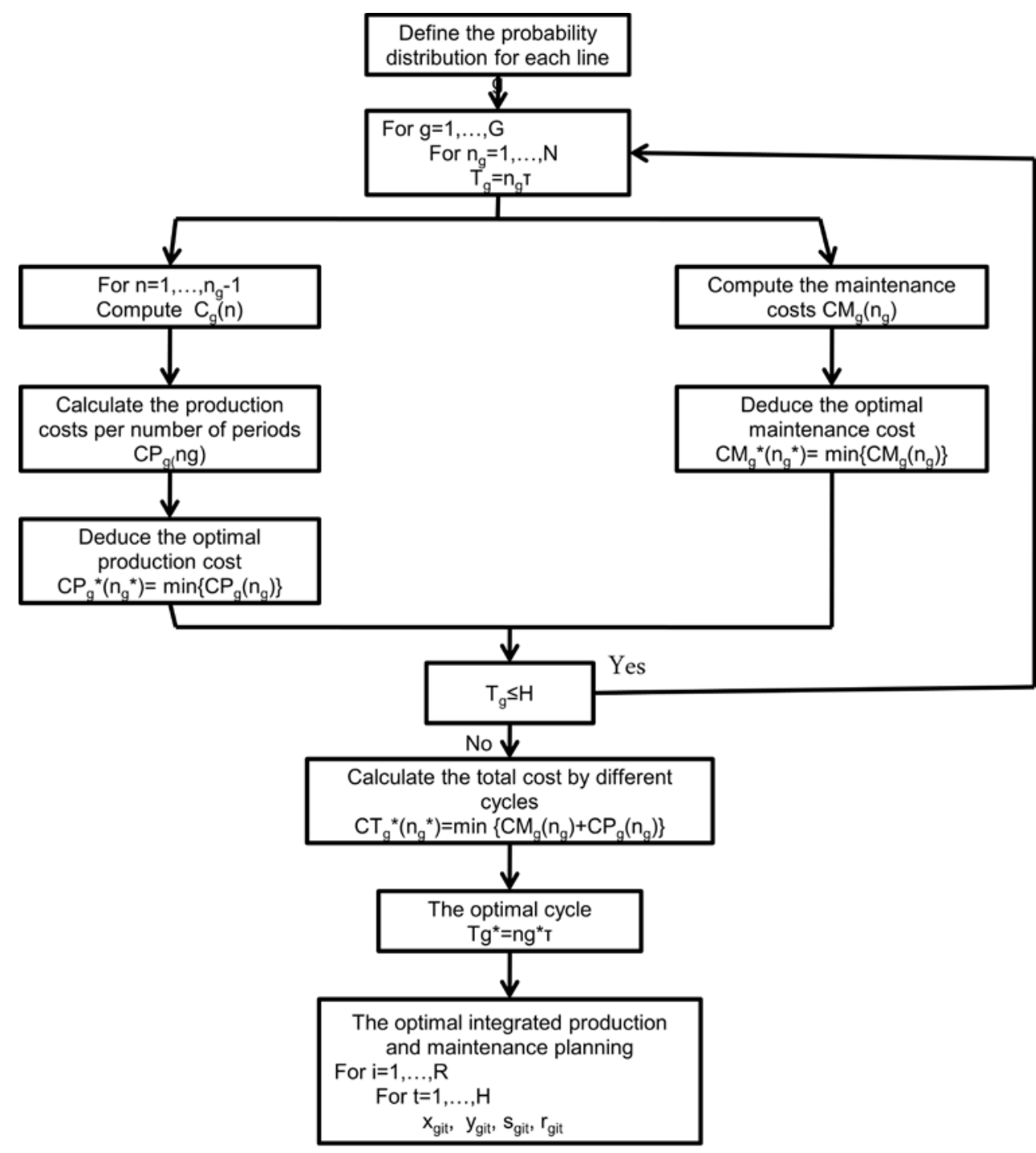

Fig. 4: Solution Algorithm Flowchart

To assess the maintenance cost we need a set of constants which are: The preventive replacement cost $c p_{1}=c p_{2}=28$ and the minimal repair action cost $c c_{1}=c c_{2}=35$ for each production line. Also it is necessary to know the failure distribution (line lifetime) that is a Gamma distribution with the parameters $(\alpha=2, \lambda=1)$ for line 1 and a Gamma distribution with the parameters $(\alpha=2, \lambda=2)$ for line 2 . 
Table 1: Demand per Product and Period

\begin{tabular}{|l|l|l|}
\hline $\begin{array}{l}\text { Demand of product i } \\
\text { in period t }\end{array}$ & Product 1 & Product 2 \\
\hline 1 & 2 & 3 \\
\hline 2 & 3 & 2 \\
\hline 3 & 2 & 3 \\
\hline 4 & 3 & 2 \\
\hline 5 & 2 & 3 \\
\hline 6 & 3 & 2 \\
\hline 7 & 2 & 3 \\
\hline 8 & 3 & 2 \\
\hline 9 & 2 & 3 \\
\hline 10 & 3 & 2 \\
\hline 11 & 2 & 3 \\
\hline
\end{tabular}

On the other hand, each basic period $\tau$ for each line has a fixed nominal capacity and its value is $C_{\max 1}=C_{\max 2}=15$. Then, every maintenance activity either preventive or corrective consumes a part of the nominal capacity $C \max g$, respectively, $P_{c 1}=P_{c 2}=5$ and $P_{p 1}=P_{p 2}=1$.

To estimate the production cost we use the production costs and the needs in resources for different products, periods and line (in our example they have the same values) as presented in Table 2.

Table 2: Production Data

\begin{tabular}{|l|l|}
\hline $\begin{array}{l}\text { Line } \\
\text { Product } \\
\text { Period }\end{array}$ & \multicolumn{1}{c|}{$\begin{array}{c}\mathrm{g}=\{1,2\} \\
\mathrm{i}=\{1,2\} \\
\mathrm{t}=\{1, \ldots, 8\}\end{array}$} \\
\hline Producing cost & 5 \\
\hline Launching cost & 25 \\
\hline Storing cost & 2 \\
\hline Breaking cost & 100 \\
\hline Fixed needs & 5 \\
\hline Variable needs & 1 \\
\hline
\end{tabular}

\section{Results and discussion}

The calculation of the maintenance costs and the capacity based on EQ3 and EQ2 respectively for the two production lines has led us to trace the two tables (Table 3 and Table 4) which recapitulate the results. Generally, the production capacity in the line decreases with the number of periods in the preventive maintenance cycle (for example, for a cycle $T_{1}=1 \tau$ the capacity is $C_{1}(1)=12.47$ this value decreases to a value 
$C_{1}(11)=10.44$ for cycle $\left.C_{1}(11)=10.44\right)$ this reduction is due to the fact that the maintenance activities consume a portion of the capacity because of the degradation of the production system with age and use.

Table 3: Maintenance Costs and Available Capacities per Period in Line 1 (Gamma $(2,1))$ in Function of the Value of the Maintenance Cycle $T_{1}=k_{1} \tau$

\begin{tabular}{|l|l|l|l|l|l|l|l|l|l|l|l|}
\hline $\mathrm{t}$ & $\mathrm{k}_{1}=1$ & $\mathrm{k}_{1}=2$ & $\mathrm{k}_{1}=3$ & $\mathrm{k}_{1}=4$ & $\mathrm{k}_{1}=5$ & $\mathrm{k}_{1}=6$ & $\mathrm{k}_{1}=7$ & $\mathrm{k}_{1}=8$ & $\mathrm{k}_{1}=9$ & $\mathrm{k}_{1}=10$ & $\mathrm{k}_{1}=11$ \\
\hline $\begin{array}{l}\mathrm{CM} \\
1\end{array}$ & 426.14 & 308.48 & 284.99 & 279.82 & 291.32 & 282.18 & 283.89 & 287.58 & 293.96 & 304.81 & 326.03 \\
\hline 1 & 12.47 & 12.47 & 12.47 & 12.47 & 12.47 & 12.47 & 12.47 & 12.47 & 12.47 & 12.47 & 12.47 \\
\hline 2 & 12.47 & 12.03 & 12.47 & 12.03 & 12.47 & 12.03 & 12.47 & 12.03 & 12.47 & 12.03 & 12.47 \\
\hline 3 & 12.47 & 12.03 & 11.44 & 12.47 & 12.03 & 11.44 & 12.47 & 12.03 & 11.44 & 12.47 & 12.03 \\
\hline 4 & 12.47 & 12.03 & 11.44 & 11.12 & 12.47 & 12.03 & 11.44 & 11.12 & 12.47 & 12.03 & 11.44 \\
\hline 5 & 12.47 & 12.03 & 11.44 & 11.12 & 10.91 & 12.47 & 12.03 & 11.44 & 11.12 & 10.91 & 12.47 \\
\hline 6 & 12.47 & 12.03 & 11.44 & 11.12 & 10.91 & 10.77 & 12.47 & 12.03 & 11.44 & 11.12 & 10.91 \\
\hline 7 & 12.47 & 12.03 & 11.44 & 11.12 & 10.91 & 10.77 & 10.67 & 12.47 & 12.03 & 11.44 & 11.12 \\
\hline 8 & 12.47 & 12.03 & 11.44 & 11.12 & 10.91 & 10.77 & 10.67 & 10.59 & 12.47 & 12.03 & 11.44 \\
\hline 9 & 12.47 & 12.03 & 11.44 & 11.12 & 10.91 & 10.77 & 10.67 & 10.59 & 10.53 & 12.47 & 12.03 \\
\hline 10 & 12.47 & 12.03 & 11.44 & 11.12 & 10.91 & 10.77 & 10.67 & 10.59 & 10.53 & 10.48 & 12.47 \\
\hline 11 & 12.47 & 12.03 & 11.44 & 11.12 & 10.91 & 10.77 & 10.67 & 10.59 & 10.53 & 10.48 & 10.44 \\
\hline
\end{tabular}

Table 4: Maintenance Costs and Available Capacities per Period in Line 2 (Gamma $(2,2))$ in Function of the Value of the Maintenance Cycle $T_{2}=k_{2} \tau$

\begin{tabular}{|l|l|l|l|l|l|l|l|l|l|l|l|}
\hline periods & $\mathrm{k}_{2}=1$ & $\mathrm{k}_{2}=2$ & $\mathrm{k}_{2}=3$ & $\mathrm{k}_{2}=4$ & $\mathrm{k}_{2}=5$ & $\mathrm{k}_{2}=6$ & $\mathrm{k}_{2}=7$ & $\mathrm{k}_{2}=8$ & $\mathrm{k}_{2}=9$ & $\mathrm{k}_{2}=10$ & $\mathrm{k}_{2}=11$ \\
\hline $\mathrm{CM}_{2}$ & 344.40 & 197.01 & 156.03 & 139.53 & 146.62 & 128.13 & 129.41 & 132.10 & 136.57 & 143.60 & 154.99 \\
\hline 1 & 13.53 & 13.53 & 13.53 & 13.53 & 13.53 & 13.53 & 13.53 & 13.53 & 13.53 & 13.53 & 13.53 \\
\hline 2 & 13.53 & 13.94 & 13.53 & 13.94 & 13.53 & 13.94 & 13.53 & 13.94 & 13.53 & 13.94 & 13.53 \\
\hline 3 & 13.53 & 13.94 & 13.62 & 13.53 & 13.94 & 13.62 & 13.53 & 13.94 & 13.62 & 13.53 & 13.94 \\
\hline 4 & 13.53 & 13.94 & 13.62 & 13.41 & 13.53 & 13.94 & 13.62 & 13.41 & 13.53 & 13.94 & 13.62 \\
\hline 5 & 13.53 & 13.94 & 13.62 & 13.41 & 13.27 & 13.53 & 13.94 & 13.62 & 13.41 & 13.27 & 13.53 \\
\hline 6 & 13.53 & 13.94 & 13.62 & 13.41 & 13.27 & 13.17 & 13.53 & 13.94 & 13.62 & 13.41 & 13.27 \\
\hline 7 & 13.53 & 13.94 & 13.62 & 13.41 & 13.27 & 13.17 & 13.09 & 13.53 & 13.94 & 13.62 & 13.41 \\
\hline 8 & 13.53 & 13.94 & 13.62 & 13.41 & 13.27 & 13.17 & 13.09 & 13.03 & 13.53 & 13.94 & 13.62 \\
\hline 9 & 13.53 & 13.94 & 13.62 & 13.41 & 13.27 & 13.17 & 13.09 & 13.03 & 12.98 & 13.53 & 13.94 \\
\hline 10 & 13.53 & 13.94 & 13.62 & 13.41 & 13.27 & 13.17 & 13.09 & 13.03 & 12.98 & 12.94 & 13.53 \\
\hline 11 & 13.53 & 13.94 & 13.62 & 13.41 & 13.27 & 13.17 & 13.09 & 13.03 & 12.98 & 12.94 & 12.90 \\
\hline
\end{tabular}


If we trace the maintenance costs for the two lines and 11 periods depending on the number of periods in cycle $\mathrm{T}_{\mathrm{g}}$ we can see that the minimum values of costs are different as well as the optimal cycles. Indeed, for the first line $C M_{1}\left(k_{1}=4\right)=279.82$ is the optimum value for the cycle $T_{1}=4 \tau$ so that preventive action must be done every 4 months, concerning the second line $C M_{2}\left(k_{2}=6\right)=128.13$ and thereafter $T_{2}=6 \tau$ is this what we can observe on Figure 5. Whereas if we do the summation of the two costs for different cases of $k_{g} \in\{1, \ldots, N\}$ in Table 3 we find that the optimal value is for a maintenance cost that is $\operatorname{CMtot}\left(k_{g}=6 \tau\right)=410.31$.

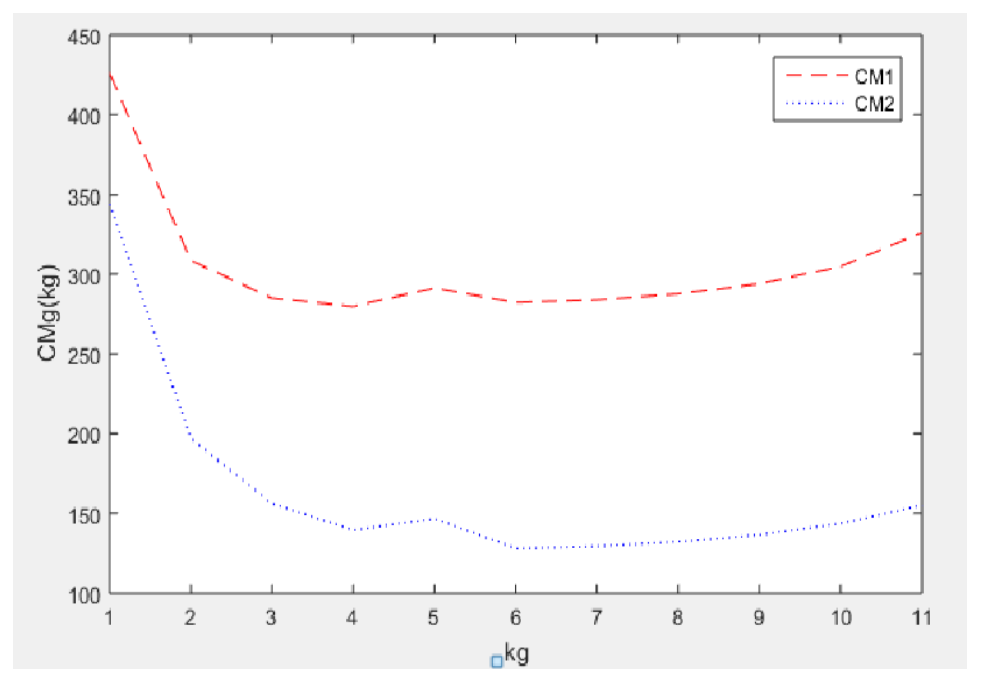

Fig. 5: Maintenance Costs in Each Line $g \in\{1,2\}$

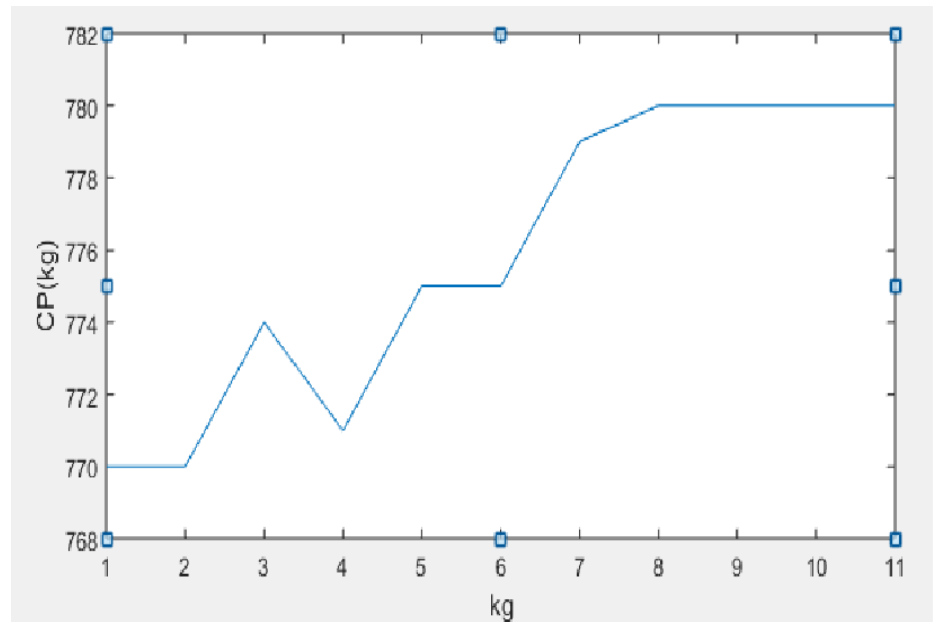

Fig. 6: The Production Cost per Number of Periods in Cycle 
On the other side, we calculate the production costs for the different cases of $T_{g}$, we get the results shown in Table 5, and we have drawn these costs depending on $\mathrm{k}_{\mathrm{g}}$. The remark that we could say is that the production costs increase with the number of periods in each maintenance cycle this is because of the decrease in capacity over time.

Table 5: Production Costs per Periods for Each Line $g \in\{1,2\}$

\begin{tabular}{|l|l|l|l|l|l|}
\hline periods & \multicolumn{1}{|c|}{ CP } & \multicolumn{1}{c|}{ CM1 } & \multicolumn{1}{c|}{ CM2 } & \multicolumn{1}{c|}{ CMtot } & Ctot \\
\hline 1 & 770 & 426.14 & 344.40 & 770.54 & 1540.5 \\
\hline 2 & 770 & 308.48 & 197.01 & 505.49 & 1275.5 \\
\hline 3 & 774 & 284.99 & 156.03 & 441.02 & 1215.0 \\
\hline 4 & 771 & 279.82 & 139.53 & 419.35 & 1190.4 \\
\hline 5 & 775 & 291.32 & 146.62 & 437.94 & 1212.9 \\
\hline 6 & 775 & 282.18 & 128.13 & 410.31 & 1185.3 \\
\hline 7 & 779 & 283.89 & 129.41 & 413.30 & 1192.3 \\
\hline 8 & 779 & 287.58 & 132.10 & 419.68 & 1199.7 \\
\hline 9 & 780 & 293.96 & 136.57 & 430.53 & 1210.5 \\
\hline 10 & 780 & 304.81 & 143.60 & 448.41 & 1228.4 \\
\hline 11 & 780 & 326.03 & 154.99 & 481.02 & 1261.0 \\
\hline
\end{tabular}

Starting from Figure 2, the optimal value of the production cost is $C P\left(k_{g}=2\right)=770$ and it is defined for $T_{g}=1 \tau$ ou $T_{g}=2 \tau$, we selected $T_{g}=2 \tau$ to minimize the number of preventive replacements. Then, we add this optimal cost to the total maintenance cost $\operatorname{CMtot}\left(k_{g}=2\right)=770.54$ for the same period and we get a total $\operatorname{cost} \operatorname{Ctot}\left(k_{g}=2\right)=1540$, this value is far away from the optimal total cost which is equal to $\operatorname{Ctot}\left(k_{g}=6\right)=1185.3$. In this case, the optimal integrated planning of maintenance and production is for preventive replacements made each $T_{g}=6 \tau$. On the other side, Table 6 represents the quantities to be produced and when to start as well as the stock and breaking on demand levels of product $i \in\{1,2\}$ in period $t \in\{1, \ldots, 11\}$ for line $g \in\{1,2\}$.

Table 6: The Optimal Integrated Planning

\begin{tabular}{|c|c|c|c|c|c|c|c|c|c|c|c|c|}
\hline$T_{g}=6 \tau$ & \multicolumn{3}{|c|}{$C P=775$} & \multicolumn{3}{|c|}{$C M t o t=410.31$} & \multicolumn{4}{|c|}{ Ctot $=1185.3$} & & \\
\hline$g \in\{1,2\}$ & \multicolumn{2}{|c|}{ Line1 } & \multicolumn{2}{|c|}{ Line 2} & \multicolumn{2}{|c|}{ Line 1} & \multicolumn{2}{|c|}{ Line 2} & \multicolumn{2}{|c|}{ Line $1 \& 2$} & \multicolumn{2}{|c|}{ Line $1 \& 2$} \\
\hline$i \in\{1,2\}$ & P1 & $\mathrm{P} 2$ & $\mathrm{P} 1$ & P2 & $\mathrm{P} 1$ & $\mathrm{P} 2$ & $\mathrm{P} 1$ & $\mathrm{P} 2$ & $\mathrm{P} 1$ & $\mathrm{P} 2$ & $\mathrm{P} 1$ & $\mathrm{P} 2$ \\
\hline periods & \multicolumn{2}{|c|}{$\mathrm{x}_{1 \mathrm{it}}$} & \multicolumn{2}{|c|}{$\mathrm{X}_{2 \mathrm{it}}$} & \multicolumn{2}{|c|}{$\mathrm{y}_{1 \mathrm{it}}$} & \multicolumn{2}{|c|}{$\mathrm{y}_{2 \mathrm{it}}$} & \multicolumn{2}{|c|}{$\mathrm{s}_{\text {it }}$} & \multicolumn{2}{|c|}{$\mathrm{r}_{\mathrm{it}}$} \\
\hline 1 & 0 & 5 & 0 & 0 & 0 & 1 & 0 & 0 & 0 & 2 & 2 & 0 \\
\hline 2 & 5 & 0 & 0 & 0 & 1 & 0 & 0 & 0 & 2 & 0 & 0 & 0 \\
\hline 3 & 0 & 5 & 0 & 0 & 0 & 1 & 0 & 0 & 0 & 2 & 0 & 0 \\
\hline 4 & 0 & 0 & 5 & 0 & 0 & 0 & 1 & 0 & 2 & 0 & 0 & 0 \\
\hline 5 & 0 & 5 & 0 & 0 & 0 & 1 & 0 & 0 & 0 & 2 & 0 & 0 \\
\hline 6 & 5 & 0 & 0 & 0 & 1 & 0 & 0 & 0 & 2 & 0 & 0 & 0 \\
\hline 7 & 0 & 7 & 0 & 0 & 0 & 1 & 0 & 0 & 0 & 4 & 0 & 0 \\
\hline 8 & 0 & 0 & 5 & 0 & 0 & 0 & 1 & 0 & 2 & 2 & 0 & 0 \\
\hline 9 & 0 & 0 & 0 & 6 & 0 & 0 & 0 & 1 & 0 & 5 & 0 & 0 \\
\hline 10 & 5 & 0 & 0 & 0 & 1 & 0 & 0 & 0 & 2 & 3 & 0 & 0 \\
\hline 11 & 0 & 0 & 0 & 0 & 0 & 0 & 0 & 0 & 0 & 0 & 0 & 0 \\
\hline
\end{tabular}




\section{Conclusion}

In this paper, we studied the problem of integrating maintenance policies with production scheduling (capacitated lot-sizing problem with setup time and cost then the breaking on demand constraint) in the context of a multi-lines system subject to preventive maintenance in the beginning of each cycle and minimal repair in case of failure. We developed a mixed integer linear programming model. The objective function is to minimize the total cost instead of planning maintenance and production separately and have more economics spending; also the contribution of the breaking on demand constraint provides the satisfaction of demands within the required delays.

An issue currently under investigation consists in studying the current model with different cases of line life time (distribution of failures), while developing and comparing some heuristic and meta-heuristics based on the obtained solution with our solution approach. As another future work, we will extend our actual model to deal with multi-line systems with one or more common resources.

\section{References}

[1]. Aghezzaf, E., Sitompul, C., Najid, N. Integrated production planning and preventive maintenance in deteriorating production systems, Information Sciences. Vol.178, No.17, pp. 3382-3392, 2008.

[2]. Yalaoui, F., Chabbi, K., Yalaoui, A., Integrated production planning and preventive maintenance in deteriorating production systems, Information Sciences. Vol.278, No.1, pp. 841-861, 2014.

[3]. [3]. Ettaye, G., El Barkany, A., El Khalfi, A., Modeling and optimization a production/maintenance integrated planning. International Journal of Engineering Research in Africa. Vol. 28, No. 1, pp. 169-181, January 2017.

[4]. Ettaye, G. A. El Barkany, A. El Khalfi, A., The integration of maintenance plans and production scheduling for a degradable multi-state system: A literature review, International journal of productivity and quality management. Vol.19, No.1, pp. 74-97, 2016.

[5]. Weinstein, L., Chung, C.H., Integrating maintenance and production decisions in a hierarchical production planning environment, Computers and Operations Research, Vol. 26, No.10-11, pp. 1059-1074, 1999.

[6]. Aghezzaf, E., Jamal, M., Ait-Kadi, D., An integrated production and preventive maintenance planning model, European Journal of Operational Research. Vol.181, No.2, pp. 679-685, 2007.

[7]. Aghezzaf, E., Najid, N., Integrated production planning and preventive maintenance in deteriorating production systems, Information Sciences, Vol.178, No.17, pp. 679-85, 2008.

[8]. Nourelfath, M., Chatelet, E., Integrating production, inventory and maintenance planning for a parallel system with dependent components, Reliability Engineering and System Safety. Vol.101, No.1, pp. 59-66, 2012.

[9]. Nourelfath, M., Fitouhi, M.C., Machani, M., An Integrated Model for Production and Preventive Maintenance Planning in Multi-State Systems, IEEE Transactions on Reliability. Vol.59, No.3, pp. 496-506, 2010.

Ghita ETTAYE is a PhD candidate at the Department of Mechanical Engineering, University of Sidi Mohammed Ben Abdellah, Morocco. She received her DEUST in physics mathematics and informatics, also a diploma of engineer in mechanical design and innovation (2013) from the same university. Her research interests include maintenance production, manufacturing system and optimization. 
Abdellah El Barkany is a Professor at the Mechanical Engineering Laboratory in Faculty of Sciences and Techniques, Sidi Mohammed Ben Abdellah University. He obtained his $\mathrm{PhD}$ in Mechanical Engineering from University Hassan II of Casablanca (2007). He received his Engineering degree from the same university in 1997. He has published many papers in different academic journals and his research interests span from design, production, quality, maintenance and optimization.

Ahmed El Khalfi is a Professor of Mechanical Engineering and Head of Mechanical Engineering Laboratory, Sidi Mohammed Ben Abdellah University. He holds his BS and $\mathrm{PhD}$ degrees in Mechanical Engineering. His areas of interest include fluid mechanics, finite element analysis, design, numeric method, quality and optimization. 